Abdullah M. Asiri*, Muhammad Nadeem Arshad, Soha M. Albukhari and Amerah M. Al-Soliemy

\title{
The crystal structure of (4Z)-2-[(E)-(1-ethyl-3,3-dimethyl-1,3-dihydro-2 $\mathrm{H}$ - indol-2-ylidene)methyl]-4-[(1-ethyl-3,3-dimethyl-3H-indolium-2-yl) methylidene]-3-oxocyclobut-1-en-1-olate, $\mathrm{C}_{30} \mathrm{H}_{32} \mathrm{~N}_{2} \mathrm{O}_{2}$
}

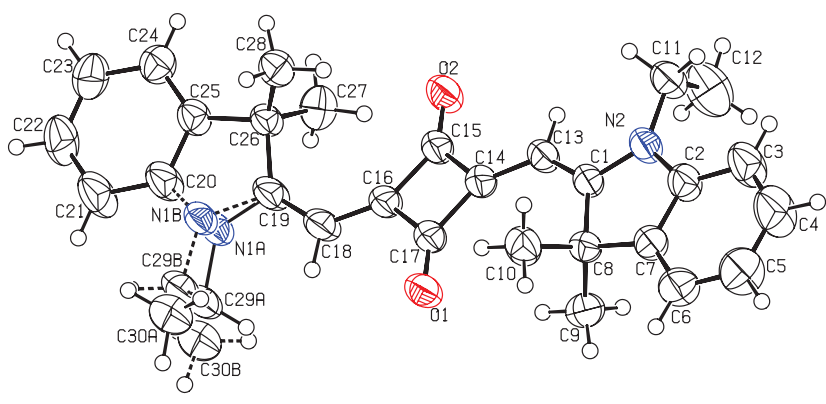

https://doi.org/10.1515/ncrs-2019-0165

Received March 4, 2019; accepted June 25, 2019; available online July 19, 2019

\begin{abstract}
$\mathrm{C}_{30} \mathrm{H}_{32} \mathrm{~N}_{2} \mathrm{O}_{2}$, monoclinic, $P 2_{1} / c$ (no. 14), $a=9.8611(2) \AA$, $b=15.8027(3) \AA, \quad c=16.2473(3) \AA, \quad \beta=100.150(2)^{\circ}$, $V=2492.23(8) \AA^{3}, Z=4, R_{\mathrm{gt}}(F)=0.0542, w R_{\text {ref }}\left(F^{2}\right)=0.1677$, $T=296(3) \mathrm{K}$.
\end{abstract}

\section{CCDC no.: 1881665}

The molecular structure is shown in the figure. Table 1 contains crystallographic data and Table 2 contains the list of the atoms including atomic coordinates and displacement parameters.

\section{Source of material}

Equimolar amount of squaric acid and 2,3,3trimethylindolenine were added to a round bottom flask

\footnotetext{
*Corresponding author: Abdullah M. Asiri, Chemistry Department, Faculty of Science, King Abdulaziz University, P.O. Box 80203, Jeddah 21589, Saudi Arabia; and Center of Excellence for Advanced Materials Research (CEAMR), King Abdulaziz University, P.O. Box 80203, Jeddah 21589, Saudi Arabia, e-mail: aasiri2@kau.edu.sa Muhammad Nadeem Arshad: Chemistry Department, Faculty of Science, King Abdulaziz University, P.O. Box 80203, Jeddah 21589, Saudi Arabia; and Center of Excellence for Advanced Materials Research (CEAMR), King Abdulaziz University, P.0. Box 80203, Jeddah 21589, Saudi Arabia

Soha M. Albukhari: Chemistry Department, Faculty of Science, King Abdulaziz University, P.O. Box 80203, Jeddah 21589, Saudi Arabia Amerah M. Al-Soliemy: Chemistry Department, Faculty of Applied Science, Umm Al-Qura University, Makkah, Saudi Arabia
}

Table 1: Data collection and handling.

\begin{tabular}{|c|c|}
\hline Crystal: & Red prismatic \\
\hline Size: & $0.32 \times 0.23 \times 0.13 \mathrm{~mm}$ \\
\hline Wavelength: & Cu $K \alpha$ radiation (1.54184 ̊̊) \\
\hline$\mu$ : & $0.59 \mathrm{~mm}^{-1}$ \\
\hline Diffractometer, scan mode: & SuperNova, $\omega$ \\
\hline$\theta_{\max }$, completeness: & $76.5^{\circ},>99 \%$ \\
\hline$N(h k l)_{\text {measured }}, N(h k l)_{\text {unique }}, R_{\text {int }}:$ & $28307,5211,0.023$ \\
\hline Criterion for $I_{\text {obs }}, N\left(h k l_{\mathrm{gt}}\right.$ : & $I_{\mathrm{obs}}>2 \sigma\left(I_{\mathrm{obs}}\right), 4340$ \\
\hline$N(\text { param })_{\text {refined }}:$ & 321 \\
\hline Programs: & $\begin{array}{l}\text { CrysAlis }^{\text {PRO }}[1] \text {, SHELX [2, 3], } \\
\text { WinGX/ORTEP [4] }\end{array}$ \\
\hline
\end{tabular}

Table 2: Fractional atomic coordinates and isotropic or equivalent isotropic displacement parameters $\left(\AA^{2}\right)$.

\begin{tabular}{lrrrr}
\hline Atom & $\boldsymbol{x}$ & $\boldsymbol{y}$ & $\boldsymbol{z}$ & $\boldsymbol{U}_{\text {iso }} / \boldsymbol{U}_{\text {eq }}$ \\
\hline O1 & $0.38808(13)$ & $0.21079(8)$ & $0.08798(7)$ & $0.0593(3)$ \\
O2 & $0.18088(15)$ & $0.03021(8)$ & $-0.11097(8)$ & $0.0643(4)$ \\
N1A & $0.4354(2)$ & $-0.11640(16)$ & $0.16244(15)$ & $0.0479(5)$ \\
N2 & $0.10553(16)$ & $0.35696(9)$ & $-0.18202(8)$ & $0.0528(4)$ \\
C1 & $0.17963(17)$ & $0.31397(10)$ & $-0.11580(9)$ & $0.0468(4)$ \\
C2 & $0.1233(2)$ & $0.44469(10)$ & $-0.17267(11)$ & $0.0548(4)$ \\
C3 & $0.0695(3)$ & $0.50780(13)$ & $-0.22790(13)$ & $0.0755(6)$ \\
H3 & 0.013143 & 0.495695 & -0.278699 & $0.091^{*}$ \\
C4 & $0.1043(3)$ & $0.58988(13)$ & $-0.20335(16)$ & $0.0917(8)$ \\
H4 & 0.072366 & 0.634167 & -0.239284 & $0.110^{*}$ \\
C5 & $0.1850(3)$ & $0.60801(12)$ & $-0.12698(16)$ & $0.0838(7)$ \\
H5 & 0.205804 & 0.663965 & -0.112118 & $0.101^{*}$ \\
C6 & $0.2351(2)$ & $0.54365(11)$ & $-0.07252(13)$ & $0.0643(5)$ \\
H6 & 0.287355 & 0.555929 & -0.020470 & $0.077^{*}$ \\
C7 & $0.20647(18)$ & $0.46094(10)$ & $-0.09646(10)$ & $0.0510(4)$ \\
C8 & $0.24897(16)$ & $0.37850(10)$ & $-0.05228(9)$ & $0.0460(4)$ \\
C9 & $0.40631(18)$ & $0.36897(12)$ & $-0.03608(13)$ & $0.0622(5)$ \\
H9A & 0.431343 & 0.316762 & -0.007141 & $0.093^{*}$ \\
H9B & 0.447220 & 0.415439 & -0.002534 & $0.093^{*}$ \\
H9C & 0.438780 & 0.368733 & -0.088412 & $0.093^{*}$ \\
C10 & $0.1902(2)$ & $0.37246(13)$ & $0.02901(11)$ & $0.0618(5)$ \\
H10A & 0.216428 & 0.319369 & 0.055891 & $0.093^{*}$ \\
H10B & 0.091560 & 0.376229 & 0.016342 & $0.093^{*}$ \\
H10C & 0.225902 & 0.417996 & 0.065610 & $0.093^{*}$ \\
C11 & $0.0249(2)$ & $0.31939(12)$ & $-0.25722(11)$ & $0.0588(4)$ \\
H11A & -0.047342 & 0.358317 & -0.281291 & $0.071^{*}$ \\
H11B & -0.018337 & 0.267792 & -0.242399 & $0.071^{*}$ \\
C12 & $0.1131(3)$ & $0.2999(2)$ & $-0.31999(15)$ & $0.0974(8)$ \\
H12A & 0.152958 & 0.351242 & -0.336406 & $0.146^{*}$ \\
H12B & 0.058211 & 0.274021 & -0.368095 & $0.146^{*}$ \\
H12C & 0.185127 & 0.261739 & -0.296057 & $0.146^{*}$ \\
C13 & $0.18389(18)$ & $0.22682(10)$ & $-0.11251(9)$ & $0.0499(4)$ \\
H13 & 0.136232 & 0.200524 & -0.160169 & $0.060^{*}$ \\
& & & &
\end{tabular}


Table 2 (continued)

\begin{tabular}{|c|c|c|c|c|}
\hline Atom & $x$ & $y$ & $z$ & $U_{\text {iso }}{ }^{*} / U_{\text {eq }}$ \\
\hline C14 & $0.24657(16)$ & $0.17051(10)$ & $-0.05082(9)$ & $0.0457(3)$ \\
\hline C15 & $0.23683(17)$ & $0.07805(10)$ & $-0.05565(10)$ & $0.0483(4)$ \\
\hline C16 & $0.31739(16)$ & $0.07047(10)$ & $0.02944(10)$ & $0.0478(4)$ \\
\hline C17 & $0.32987(16)$ & $0.16301(10)$ & $0.03328(10)$ & $0.0464(3)$ \\
\hline C18 & $0.37367(19)$ & $0.01451(11)$ & $0.09213(11)$ & $0.0595(5)$ \\
\hline H18 & 0.435702 & 0.039208 & 0.135174 & $0.071^{*}$ \\
\hline C19 & $0.35424(19)$ & $-0.07125(11)$ & $0.10218(11)$ & $0.0585(5)$ \\
\hline C20 & $0.3714(2)$ & $-0.19788(11)$ & $0.17147(12)$ & $0.0665(5)$ \\
\hline C21 & $0.4087(3)$ & $-0.25950(13)$ & $0.23240(14)$ & $0.0813(7)$ \\
\hline $\mathrm{H} 21$ & 0.475742 & -0.249589 & 0.279298 & $0.098^{\star}$ \\
\hline C22 & $0.3409(3)$ & $-0.33633(13)$ & $0.21961(14)$ & $0.0747(6)$ \\
\hline $\mathrm{H} 22$ & 0.361706 & -0.378803 & 0.259452 & $0.090^{*}$ \\
\hline C23 & $30(2)$ & $-0.35156(12)$ & $0.14922(14)$ & $0.0685(5)$ \\
\hline $\mathrm{H} 23$ & 0.200635 & -0.404249 & 0.141643 & 0.082 * \\
\hline C24 & $0.2075(2)$ & $-0.28841(11)$ & $0.08957(12)$ & $0.0577(4)$ \\
\hline $\mathrm{H} 24$ & 0.141319 & -0.298389 & 0.042271 & $0.069^{\star}$ \\
\hline $\mathrm{C} 25$ & $0.27176(17)$ & $-0.21115(10)$ & $0.10163(10)$ & $0.0491(4)$ \\
\hline C26 & $0.25535(16)$ & $-0.13156(9)$ & $0.04898(9)$ & $0.0445(3)$ \\
\hline $\mathrm{C} 27$ & $0.3007(2)$ & $-0.14769(12)$ & $-0.03531(12)$ & $0.0616(5)$ \\
\hline $\mathrm{H} 27 \mathrm{~A}$ & 0.297568 & -0.095606 & -0.066003 & 0.092 * \\
\hline H27B & 860 & -0.1 & -0.06 & $0.092^{\star}$ \\
\hline $\mathrm{H} 27 \mathrm{C}$ & 0.393036 & -0.169450 & -0.025821 & 0.092 * \\
\hline C28 & $0.10702(17)$ & $-0.09921(11)$ & $0.03629(11)$ & $0.0545(4)$ \\
\hline $\mathrm{H} 28 \mathrm{~A}$ & 0.083609 & -0.084940 & 0.089493 & $0.082^{\star}$ \\
\hline $\mathrm{H} 28 \mathrm{~B}$ & 0.045832 & -0.142477 & 0.010161 & $0.082^{\star}$ \\
\hline $\mathrm{H} 28 \mathrm{C}$ & 0.098487 & -0.049893 & 0.001256 & $0.082^{\star}$ \\
\hline$C 29 A^{a}$ & $0.5472(3)$ & $-0.0827(2)$ & $0.22519(18)$ & $0.0605(7)$ \\
\hline $\mathrm{H} 29 \mathrm{~A}^{\mathrm{a}}$ & 0.597763 & -0.040594 & 0.199395 & $0.073^{\star}$ \\
\hline$H 29 B^{a}$ & 0.610406 & -0.128103 & 0.245719 & $0.073^{\star}$ \\
\hline$C 30 A^{a}$ & $0.4948(4)$ & $-0.0439(3)$ & $0.2962(2)$ & $0.0833(9)$ \\
\hline $\mathrm{H} 30 \mathrm{~A}^{\mathrm{a}}$ & 0.570732 & -0.022582 & 0.336039 & $0.125^{\star}$ \\
\hline $\mathrm{H} 30 \mathrm{~B}^{\mathrm{a}}$ & 0.433540 & 0.001715 & 0.276270 & $0.125^{\star}$ \\
\hline $\mathrm{H} 30 \mathrm{C}^{\mathrm{a}}$ & 0.446174 & -0.085729 & 0.322559 & $0.125^{\star}$ \\
\hline$C 29 B^{b}$ & $0.4819(6)$ & $-0.0757(4)$ & $0.2561(4)$ & $0.0605(7)$ \\
\hline $\mathrm{H} 29 \mathrm{C}^{\mathrm{b}}$ & 0.455893 & -0.017540 & 0.264582 & $0.073^{\star}$ \\
\hline$H 29 D^{b}$ & 0.472066 & -0.107335 & 0.305894 & $0.073^{\star}$ \\
\hline $\mathrm{C} 30 \mathrm{~B}^{\mathrm{b}}$ & $0.6258(7)$ & $-0.0775(5)$ & $0.2466(4)$ & $0.0833(9)$ \\
\hline$H 30 D^{b}$ & 0.681767 & -0.053737 & 0.295443 & $0.125^{\star}$ \\
\hline $\mathrm{H} 30 \mathrm{E}^{\mathrm{b}}$ & 0.653406 & -0.134968 & 0.239678 & $0.125^{\star}$ \\
\hline $\mathrm{H} 30 \mathrm{~F}^{\mathrm{b}}$ & 0.637214 & -0.045067 & 0.198317 & $0.125^{\star}$ \\
\hline$N 1 B^{b}$ & $0.3866(5)$ & $-0.1107(3)$ & $0.1847(3)$ & $0.0479(5)$ \\
\hline
\end{tabular}

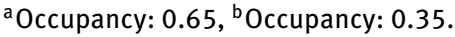

containing toluene $(20 \mathrm{~mL})$ and connected with Dean-Stark apparatus. The mixture was refluxed for $20 \mathrm{~h}$. The progress was monitored using thin layer chromatographic (TLC) technique. Upon reaction completion, the mixture was cooled to room temperature, which provided dark red crystaline material. The product was washed with petroleum ether and dried.

\section{Experimental details}

Data collection was accomplished using CrysAlis ${ }^{\mathrm{PRO}}$ software [1] at $296 \mathrm{~K}$ using $\mathrm{Cu} \mathrm{K \alpha}$ radiation. Structure solution was performed using SHELXS-2016/6 [2] and refined by fullmatrix least-squares methods on $F^{2}$ using SHELXL-2016/6 [3], in-built within WinGX [4]. All non-hydrogen atoms were refined anisotropically. The figure was generated through ORTEP [4]. All the aromatic C-H hydrogen atoms were positioned geometrically with $\mathrm{C}-\mathrm{H}=0.93 \AA$ and treated as riding atoms with $U_{\text {iso }}(\mathrm{H})=1.2 U_{\text {eq }}(\mathrm{C})$. Methyl and methylene hydrogen atoms were also positioned geometrically with $\mathrm{C}-\mathrm{H}=0.96 \AA$ for methyl and $\mathrm{C}-\mathrm{H}=0.97 \AA$ for methylene and treated as riding atoms where $U_{\text {iso }}(\mathrm{H})$ was set to $1.5 U_{\text {eq }}(\mathrm{C})$ for methyl and 1.2 $U_{\text {eq }}(\mathrm{C})$ for methylene hydrogen atoms.

\section{Comment}

Since the first report appeared by Von Sprenger and Ziegenbein [5], bis(indolenine)squaraine based molecules have been studied for their remarkable fluorescence activities $[6,7]$. There are many reports favouring these matarials for sensing protein molecules $[8,9]$. Smith and Lynch reported the parent dye 2,4-[(3,3-dimethyl-2-indolylidene)methyl] cyclobutenediylio-1,3-diolate with two chloroform solvent molecules [10]. Natsukawa and Nakazumi reported the crystal structure of the methanol solvate of the title compound [11]. In the crystal structure of the title compound (cf. the figure), two indole ring systems (C1-C8/N1) \& (C19-C26/N2) are attached to the oxo-cyclobutene moiety. The ambient-temperature data indicates the presence of disorder, over two positions, in both $\mathrm{N}$-ethyl functional groups, which was successfully modelled for one of the two groups. The N-ethyl group (N2/C29/C30), was refined using the restraints and constraints with the occupancy ratio fixed to $0.65: 0.35$ for $(\mathrm{N} 2 \mathrm{~A} / \mathrm{C} 29 \mathrm{~A} / \mathrm{C} 30 \mathrm{~A})$ and (N2B/C29B/C30B) parts, respectively. The disorder involving $\mathrm{N} 2$ produced two different planes containing the atoms (C19-C26/N2A) and (C19-C26/N2B). In the literature, the structures reported by Smith and Lynch [10] and by Natsukawa and Nakazumi [11] both have a plane of symmetry passing through the cyclobutene ring, while there is no plane symmtry in the structure of title compound. The root mean square deviation values for the planes produced from the fitted atoms belonging to the indole rings are $0.0184(2) \AA, 0.0624(2) \AA$ and $0.1129(2) \AA$ for the (C1-C8/N2), (C19-C26/N2A) and (C19-C26/N2B), respectively. The central cyclobutene ring is oriented at dihedral angles of $2.651(3)^{\circ}, 19.647(6)^{\circ}$ and $14.657(3)^{\circ}$ with respect to the (C1-C8/N2), (C19-C26/N2A) and (C19-C26/N2B) rings. These values indicate that this dye molecule is nonplanar. We have observed two non-classical hydrogen-bonding interactions which connect molecules to generate a two-dimensional network along the diagonal of the ac-plane.

Acknowledgements: This paper was funded by the Deanship of Scientific Research (DSR) at King Abdulaziz University, Jeddah, under grant no. RG-03-102-428. The authors, therefore, acknowledge with thanks DSR for technical and financial support. 


\section{References}

1. Agilent Technologies: CrysAlis ${ }^{\mathrm{PRO}}$. Agilent Technologies, Yarnton, England (2012).

2. Sheldrick, G. M.: SHELXT - integrated space-group and crystalstructure determination. Acta Crystallogr. A71 (2015) 3-8.

3. Sheldrick, G. M.: Crystal structure refinement with SHELXL. Acta Crystallogr. C71 (2015) 3-8.

4. Farrugia, L. J.: WinGX and ORTEP for Windows: An update. J. Appl. Crystallogr. 45 (2012) 849-854.

5. Von Sprenger, H.-E.; Ziegenbein, W.: Das Cyclobuten-diyliumKation, ein neuartiger Chromophor aus Quadratsäure. Angew. Chem. 79 (1967) 581-582.

6. Khopkar, S.; Deshpande, S.; Shankarling, G.: Greener protocol for the synthesis of NIR fluorescent indolenine-based symmetrical squaraine colorants. ACS Sustainable Chem. Eng. 6 (2018) 10798-10805.

7. Nizomov, N.; Ismailov, Z. F.; Nizamov, S. N.; Salakhitdinova, M. K.; Tatarets, A. L.; Patsenker, L. D.; Khodjayev, G.:
Spectral-luminescent study of interaction of squaraine dyes with biological substances. J. Mol. Struct. 788 (2006) 36-42.

8. Volkova, K. D.; Kovalska, V. B.; Tatarets, A. L.; Patsenker, L. D.; Kryvorotenko, D. V.; Yarmoluk, S. M.: Spectroscopic study of squaraines as protein-sensitive fluorescent dyes. Dyes Pigments 72 (2007) 285-292.

9. Oswald, B.; Patsenker, L.; Duschl, J.; Szmacinski, H.; Wolfbeis, O. S.; Terpetschnig, E.: Synthesis, spectral properties, and detection limits of reactive squaraine dyes, a new class of diode laser compatible fluorescent protein labels. Bioconjugate Chem. 10 (1999) 925-931.

10. Smith, D.; Lynch, D. E.: 2-[(3,3-Dimethylindolin-2-ylidene) methyl]-4-[(3,3-dimethyl-3H-indol-1-ium-2-yl)methylidene]-3oxocyclobut-1-en-1-olate chloroform disolvate. Acta Crystallogr. E69 (2013) 0786-0787.

11. Natsukawa, K.; Nakazumi, H.: Sangyo Gijutsu Sogo Kenkyujo Hokuku 6 (1993) 16. 